\title{
Anti-Hedonistic Mechatronic Systems
}

\author{
Lorenzo Scalera, Paolo Gallina, Alessandro Gasparetto and Marco Giovagnoni
}

\begin{abstract}
In this paper the concept of anti-hedonistic mechatronic systems interacting with humans is discussed. Up to this time, people have used their creativity to design machines which could reduce human efforts (i.e. robots) or enhance the perceived pleasure (i.e. entertainment tools and virtual reality systems). Nowadays, new machines designed to prevent people from doing something are emerging. Examples are: intragastric balloons to prevent people from eating, timed cigarettes boxes to prevent people from smoking, bracelets to prevent people from nail biting, alcohol-testers connected to car starter to avoid driving under alcohol influence.

The aim of this work is to present a survey about anti-hedonistic machines, providing general definitions and a possible classification. In particular a mechatronic system designed to motivate users to do push-ups exercises, by controlling the television energy supply, is presented.
\end{abstract}

Key words: Anti-Hedonistic Machine, mechatronic systems, Human-Machine Interaction, precommitment, biomechanics

\section{Introduction}

In the field of Human-Machine Interaction (HMI), the term anti-hedonistic machines (AHMs) refers to a new kind mechatronic systems designed to prevent people from feeling immediate pleasure. In other words, AHMs should be able to limit or inhibit the chance to reach an immediate and instinctive pleasure.

Every AHM needs the user to be aware about the aim of the machine itself before

Lorenzo Scalera, Alessandro Gasparetto and Marco Giovagnoni

University of Udine, e-mail: scalera.lorenzo@spes.uniud.it, alessandro.gasparetto@uniud.it, marco.giovagnoni@uniud.it

Paolo Gallina

University of Trieste, e-mail: pgallina@units.it 
starting to use it. The initial awareness is fundamental for the correct functionality and efficiency of such a machine. In fact, people using AHMs need to know that, in a short time, their willpower would weaken and have to accept in advance the deprivation effects and the discomfort conditions which the machine would cause to them [5], [6].

This concept could be explained through the myth of Ulysses. According to the myth, the greek warrior ordered to the crew to be tied to the mast when his mind was still clear, in order to avoid to succumb to siren flatteries when his mind would have been benighted. Another example could be taken from italian literature. It is known the firm decision of Vittorio Alfieri, who ordered to his servant to be tied to his chair for the purpose of finish the tragedy he was writing as soon as possible. The playwright in fact kept putting off the tragedy writing because of his attractions for other pleasure situations [4]. In both Ulysses's and Alfieri's example it is possible to identify the ropes as a rudimental but efficient example of AHM.

Taking advantage of an artefact planned in the present to modify or restrict someone's freedom of choice in the future is a concept that attracted considerable interest in the last few years, in particular referring to the notion of precommitment [5], [6], [16]. Using an AHM could be read as the outsourcing of a person own willpower: to delegate out of the mind choices which could be advantageous in the long future.

The main contribution of this paper is the definition of AHMs, providing the main operating principle and a possible classification. The concept is discussed for a real scenario, namely a mechatronic system designed to motivate users to do push-ups exercises based on precommitment concept.

\section{Operating principles}

To better understand AHMs operating principles first of all it is necessary to consider that every time someone have to make a choice, he assigns a value to all the possible alternatives he has. In most cases the perceived value associated to a reward decades according to the delay with which it is possible to benefit from the reward [11], [15]. This concept is explained in Fig. 1(a). The horizontal axis indicates the reward delay and the vertical one shows the amount of value perceived for that reward. The more the delay increases, the more the perceived value is affected by a higher temporal discount. In literature curves that model the perceived value decay are characterised by an exponential or hyperbolic trend [1].

If an artefact or a machine interacts with someone to change his own choices and make the precommitment effective, not only the temporal discount but also the costs, which the subject have to pay to reach the reward, should be considered [14].

For example, going to the gym is for many people a way to gain an excellent physical condition, which is the reward pursued by them in the long term. The value perceived from this reward is not constant over time but it is subjected to a temporal discount. In fact, if only it was enough to do a single exercise to gain the perfect physical condition, the value perceived from the reward would be great. On the other 


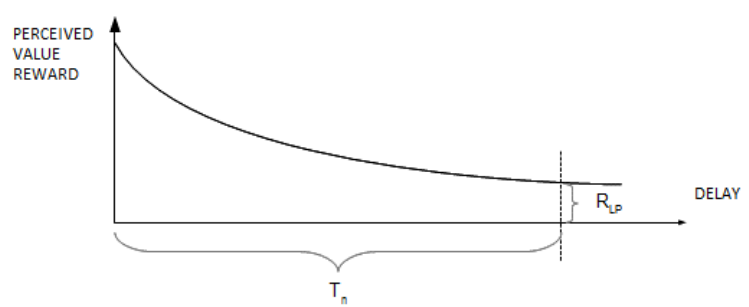

(a) Perceived value reward over time.

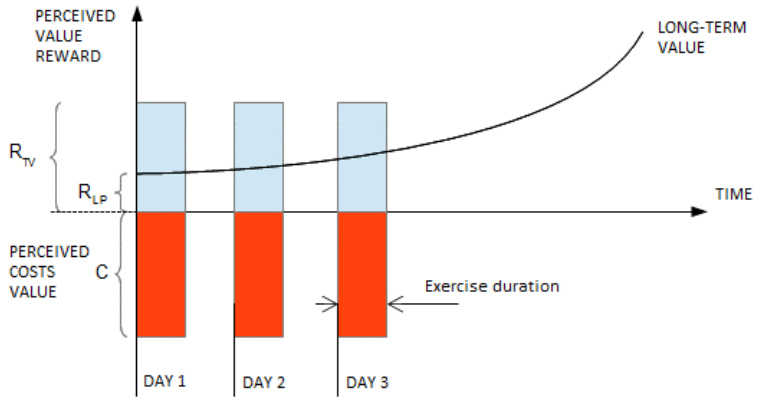

(b) Perceived value reward, costs and machine reward over time.

Fig. 1: Graphical visualization of temporal discounting concept.

side, if it was need to do exercises for years this value would definitely decrease. In particular, the de-growth of this value is proportional to the delay with which the result would appear.

For further explain AHMs role in precommitment scheme, the symmetrical to the vertical axis curve of temporal discounting is used (Fig. 1(b)). In this case, the value $R_{L P}$ in $t=0$ represents the discounted value of the reward for a delay equal to $T_{n}$, which is to the value assigned to the perfect physical condition.

Doing physical exercises involves a considerable amount of energy and a sort of discomfort that becomes a cost in the amount of motivations needed to take a decision. The cost $C$ contrasts the motivational effect of the reward. As it is shown in Fig. 1(b), this cost is constant during the exercise and is null after this one. The figure shows that at the beginning costs are higher than the long term reward.

$$
R_{L P}+C<0
$$

This inequality can explain that, if costs are higher than the perceived reward, the exercise would not be done.

The mechatronic system presented in Sect. 4 operates right at this stage, introducing a third contribute to Eq. (1). Having recognised the correct execution of the exercise (a set of push-ups in the presented case), the machine could allow the switching on of the television by means of control system. If instead the exercise has 
not been executed, the television will not be switched on. The possibility of watching television is to be considered a third reward, named machine reward. This last is indicated with $R_{T V}$ and, for sake of simplicity, it could be considered a constant during the exercise execution (as the matter of fact $R_{T V}$ could change over time). The machine reward increases the one related to the perfect physical condition. Finally, it is possible to conclude that someone would execute push-ups exercises only in the event in which Eq. (2) is verified.

$$
R_{T V}+R_{L P}+C>0
$$

In Sect. 3 a classification of different types of artefacts and mechatronic systems, which induce or drive away someone to do an action, is proposed.

\section{Classification of Anti-Hedonistic Machines}

AHMs can operate in large variety of modalities, introducing a reward or a deterrent which could persuade someone from misbehaving. In Fig. 2 a classification (nonexhaustive) of these machines and systems is proposed.

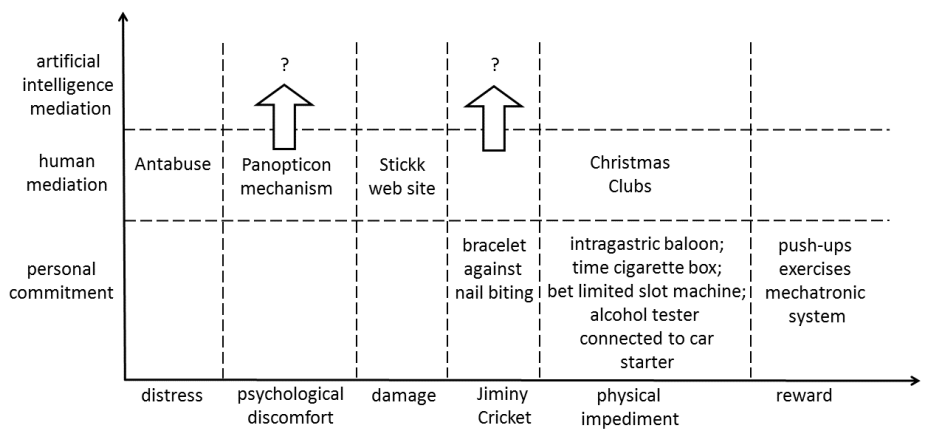

Fig. 2: Anti-hedonistic machines classification matrix.

The matrix columns indicate the manner in which AHMs operate: from a motivational reward to a inhibitory high cost, such as psychological discomfort or distress. The rows show the different ways in which an AHM could be implemented: autonomously, with human mediating, up to an artificial intelligence (AI) support.

To this regard, AI agents, such as chat-bots or artificial personal assistants, could play a significant role in preventing undesired behaviours.

The push-ups exercises mechatronic system, integrated with television control, introduces a reward and does not need an external human mediator to operate. It is positioned in the right-bottom side of the matrix. 
A machine for physical exercises could also be integrated with a software able to modify the present body shape of the user, virtually showing the benefit of a long term exercise training on his body [7]. This is a sort of virtual reward.

A second class of AHMs is based on the concept of physical impediment. In this category, intragastric balloon, time cigarette box, bet limited slot machine and alcohol tester connected to car starter could be found. The first is a technology developed to treat obesity and it consists in a balloon which partially fills the stomach and limits the food intake [8]. The intragastric balloon certainly constitutes an AHM developed to recover the imbalance caused by evolutionary mismatch: the mismatch between hereditary features of individuals, which are adapted for a past resourcepoor environment, and the present context, unfit for that hereditary features [9], [12], [18].

The second artefact is a small box which prevents people from immoderately smoking, providing cigarettes only after a pre-set timing. Obviously, it is not possible to damage this kind of machine without introducing another cost.

Bet limited slot machines are a deterrent for pathological gambling, presetting the maximum upper game limit above which it is not more possible to bet [13].

Another impediment-based mechanical system is an alcohol-tester connected to car starter: before playing the engine it is necessary to blow in a pipe and if the detected alcohol level is higher than the permitted one, the car do not start.

In the same column, but in the "human mediation" row, it is possible to find an example of bank transfer which can be considered as an AHM. Christmas Club were deposit funds very popular in the USA during the recession of the 1930s. Every week a tiny amount of money was transferred on the fund and the total amount would be given back only during Christmas time [17].

On the left column Jiminy Cricket concept based machines could be found. The cricket acted as Pinocchio's conscience; in the same manner, machines belonging to this category are reminders for people using them. An example is given by special non-removable bracelets which prevent people from nail biting [10].

Moving to the left in the matrix, AHMs developed for strong and persuasive actions can be found. Because of the potential dangerous impact of the machine, without the mediation of specialist staff, the "personal commitment" box in this column is empty. www.stickk.com is a website in which it is possible to publish a personal commitment. At the same time the user has to transfer to the website an amount of money, which would not be given back if the goal has not been reached [2].

Psychological discomfort-based machines operate by means of the panopticon mechanism [3]. The term means "to monitor everything" and it refers to the ethical and correct behaviour of someone who knows to be under control. Lifelogging is an example of this group of emerging technologies which include all wearable sensors enabled to collect visual and biometric informations about a person and make them available to community through social networks.

On the far left column of the matrix distress-based machines are allocated. Disulfiram, better known as Antabuse, is a medicine used in the treatment of chronic alcoholism by producing the effects of a "hangover" if alcohol is consumed. It works 
as a real AHM which uses a chemical deterrent to prevent people from drinking alcohol. Human mediating, as the doctor who prescribes it, is strictly required.

\section{Push-ups exercises mechatronic system}

In the present section a push-ups exercises mechatronic system is presented.

As described in Sect. 2, this machine induces the user in doing push-ups introducing a reward (indicated with $R_{T V}$ in Eq. (2)): the possibility of watching television if the exercises have been correctly done; or a deterrent: the impossibility of switching on the lighting or powering the fridge. In Fig. 3 an operating scheme of the AHM is shown.
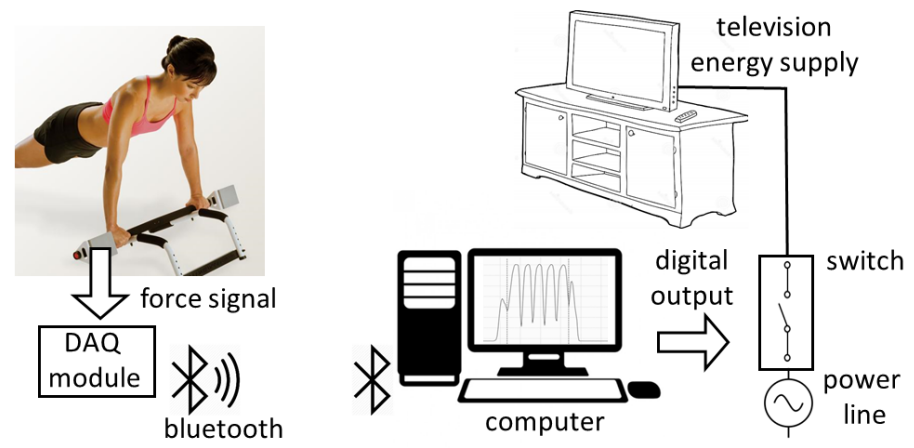

Fig. 3: Anti-hedonistic mechatronic system operating scheme.

The mechatronic prototype is composed by a gym push-ups tool, on which a measurement set-up is installed, a data acquisition system and a control device which activates the television energy supply. Data from fitness exercises are provided by two VISHAY BAK 200 strain gauges mounted on the metal bar of the tool, which are able to detect the bar deformation during the exercises.

The bar displacement is traduced into a voltage signal by means of strain gauges, which are integrated in a full Wheatstone bridge. This analogue signal is filtered and converted into a digital one in a EMANT 380 DAQ module and it is sent to a computer by means of a Bluethooth connection. The signal is analysed by a control software implemented in Python environment and based on a simple machine learning algorithm, which compares each training session with a reference one. This last has to be executed in a proper manner in the initial phase, when the user is determined and his willpower still strong. After the signal analysis, the control system returns in output a boolean variable which closes or opens a switch depending on the outcome of the check. If the exercises are correctly executed, the switch will be closed and the television could be switched on. If no, the digital output consists in 
a false constant and the power line will be interrupted. Furthermore, the system is programmed in a way that it requires a daily amount of exercises. In Fig. 4 a picture of the push-ups tool is shown. On the left-back side the DAQ module can be seen and, on the right side, the control system box with the switch of the 220 Volt electric current, is reported. The mechanical system has been developed in such a way to prevent the user from easily bypassing the switch.

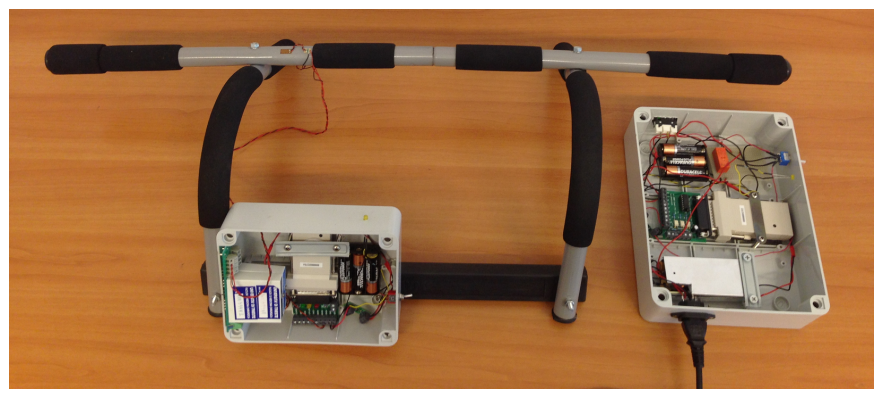

Fig. 4: Push-ups exercises mechatronic system.

Fig. 5 shows a graphical representation of strain gauges signal trend versus time during a push-ups exercise, related to an execution of five push-ups. The lowest points in the graph are related to the moments in which the slope of the push begins, while the peaks are correlated to the beginning of the rise, when the bending moment is maximum. As it can be seen from the graph, two fictitious peaks, the first and the last one, are present. These two are correlated to the load stage, in which the user grasps the tool with his hands, and the discharge phase, moment in which the exercise is finished. This two fictitious peaks have to be necessarily discarded. Therefore, the signal compared with the reference one is only the section included between the two red and dotted vertical lines.

\section{Conclusions}

In this paper the concept of anti-hedonistic machines interacting with humans has been proposed. A general definition and the operating principles of this new technologies have been provided and, in particular, the concepts of precommitment, temporal discounting, cost and machine reward have been investigated.

Different examples have been discussed and a classification (non-exhaustive) of these machines has been proposed. In this context, the manners in which artefacts operate and the ways in which they could be implemented have been exposed.

To validate the illustrated concept, a prototype of anti-hedonistic mechatronic system, designed to motivate users to do fitness exercises by controlling the television energy supply, has been developed. 


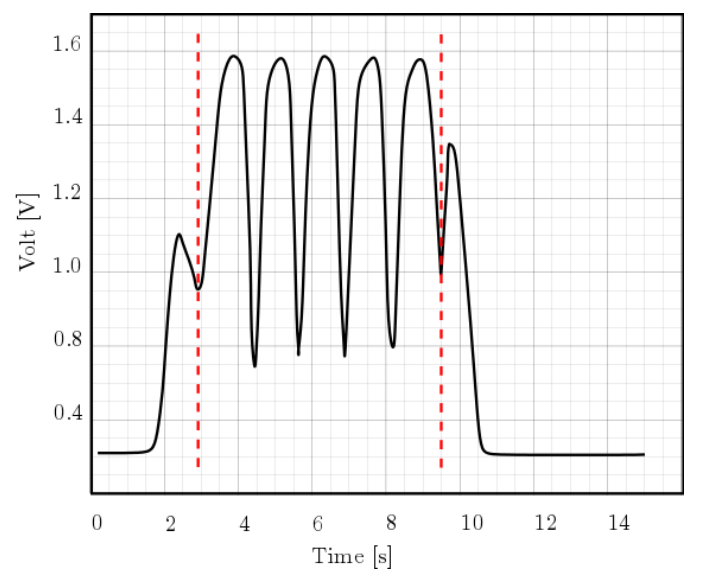

Fig. 5: Graphical representation of voltage trend during push-ups exercise.

\section{References}

1. Ainslie G. (1992). Picoeconomics: the strategic interaction of successive motivational states within the person. New York: Cambridge University.

2. Appel, J. e Karlan, D. (2011). More Than Good Intentions. Dutton Press, pp. 160-161.

3. Bentham, J. (1995). The Panopticon Writings. Ed. Bozovic, M., London.

4. D'ambra L. (1938). Il trageda legato alla sedia. Zanichelli Editore.

5. Elster J. (1979). Ulysses and the sirens. Cambridge, Cambridge University Press.

6. Elster J. (2000). Ulysses unbound. Cambridge, Cambridge University Press.

7. Fox, J. e Bailenson, J.N. (2009). Virtual self-modeling: The effects of vicarious reinforcement and identification on exercise behaviors. Media Psychology. 12, pp. 1-25.

8. Göttig, S., Weiner, R.A. e Daskalakis, M. (2009). Preoperative weight reduction using the intragastric balloon. Obesity Facts, 2, pp. 20-23.

9. Kelley, A. E., e Berridge, K. C. (2002). The neuroscience of natural rewards: Relevance to addictive drugs. Journal of Neuroscience. 22, pp. 3306-3311.

10. Koritzky G. e Yechiam E. (2011). On the value of nonremovable reminders for behavior modification: an application to nail-biting (onychophagia). Behavior modification, 35, pp. 511-30.

11. Mazur, J. E. (1997). Choice, delay, probability, and conditioned reinforcement. Animal Learning and Behavior. 25, pp. 131-147.

12. Nesse, R. M., e Berridge, K. C. (1997). Psychoactive drug use in evolutionary perspective. Science 278, pp. 63-66.

13. Nower, L. e Blaszczynski, A. (2010). Gambling Motivations, Money-Limiting Strategies and Precommitment Preferences of Problem Versus Non-Problem Gamblers. Journal of Gambling Studies. 26, pp. 361-372.

14. Paglieri F. (2013). The costs of delay: waiting versus postponing in intertemporal choice. Journal of experimental analysis of behavior. 99, pp. 362-77.

15. Raiff, B. R. e Yoon, J. (2010). From bench to bedside: A review of Impulsivity: The behavioral and neurological science of discounting. Behavioural Processes. 84, pp. 632-633.

16. Schelling, T. C. (1966). Arms and Influence. New Haven, CN: Yale University Press.

17. Strotz R. H. (1955). Myopia and inconsistency in dynamic utility maximization. The Review of Economic Studies. 23, pp. 165-180.

18. Tooby, J., e Cosmides, L. (1990). The past explains the present: Emotional adaptations and the structure of ancestral environments. Ethology and Sociobiology. 11, pp. 375-424. 